\title{
Reactivity Studies of Thiophosgene and its Halo Versions towards Diazo Derivatives
}

\author{
Sudershan R. Gondi * \\ Department of Chemistry, Southern Methodist University, 3215 Daniel
}

Avenue, Dallas, Texas 75275-0314, USA

\begin{abstract}
:
The reactions of thiophosgene with diazo derivatives gives dichloro-alkene derivatives and cyclized, 1,2,3-thiadiazoles respectively. The product formation is mainly depending on substitutions on diazo substrates. When its halo versions, $\mathrm{CSBr}_{2}$ and in combination with bromide ion, $\mathrm{CSBr}_{2}$ less reactive than bromide ion with disubstituted diazo, give gemdihalo derivatives whereas more reactive with mono substituted diazo to gives $1,2,3-$ thiadiazoles. In case $\mathrm{CSI}_{2}$ irrespective of substitution on diazo, iodide ion is more reactive then $\mathrm{CSI}_{2}$ was observed.
\end{abstract}

\section{Key words:}

Diazo acetate, Thiophosgene, gem-Dihalo, Diazirine, 1,2,3-Thiadiazoles.

\section{Introduction:}

Synthesis of tetra substituted alkenes by using thiocarbonyl and diazo were reported in the literature ${ }^{1-2}$. We are particularly interest, the combination of thiophosgene with diazo derivatives, the compounds obtained by these combinations are thioether ${ }^{3}$, thiazolines ${ }^{4-}$ 
${ }^{5}$ dihalo alkene ${ }^{6}$, thiocarbonyl chlorides ${ }^{7}$, thioisocyate ${ }^{8}$ and thiourea ${ }^{9}$. Dihalo alkene also reported by using $\mathrm{CCl}_{4}$ under anhydrous conditions ${ }^{10}$.

\section{Present work:}

We are interested in the synthesis of dihalo alkenes because it is usefulness for converting into active intermediates ${ }^{11-13}$ by using thiophosgene and its halo versions $\left(\mathrm{CSBr}_{2}\right.$ or $\left.\mathrm{CSI}_{2}\right)$. To test this, we treated various substrates

Scheme-1

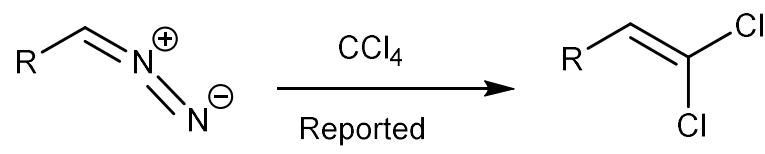

Frist when thiophosgene was treated with 1 a disubstituted diazo ${ }^{14}$, we obtained dichloro alkene $\mathbf{2} \mathbf{a}$ as major product along with impurities, and its beta lactam amide bond cleaved product $3 \mathbf{a}$ as minor product. The $3 \mathbf{a}$ is obtained due the $\mathrm{C}_{5}=\mathrm{N}$ bond formation followed by amide bond cleavage. The aldehyde peak in NMR Is consistent with predicted values. in FT-IR shows Isothiocyanate peak at $2060 \mathrm{~cm}^{-1}$, which can be formed by :S-C=N enolization ${ }^{15}$ The mass $\mathrm{m} / \mathrm{s}$ shows 447.2 peaks. (scheme-2)

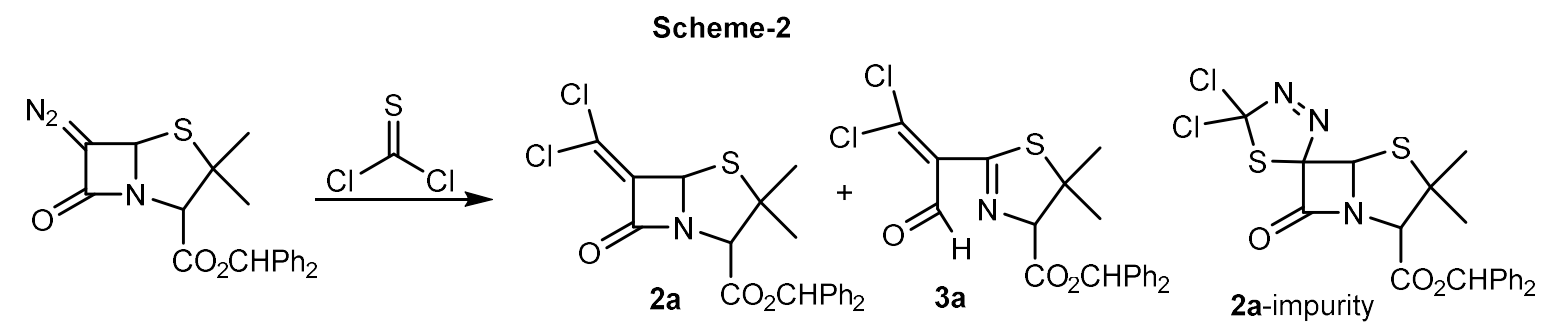

On repeating the reaction with pyridine diazo $\mathbf{1 b}$, we isolated a product $\mathbf{2 b}$ it contains, the C13-NMR values consistent with product and the characteristics diazo peak in FTIR shows at $2240 \mathrm{~cm}^{-1}$ is absent in product $\mathbf{3 a}$. The formation of $\mathbf{3 b}$ is rules out based on C13-NMR, where gem-dichloro peaks shows at 95 ppm. (Scheme-3) 


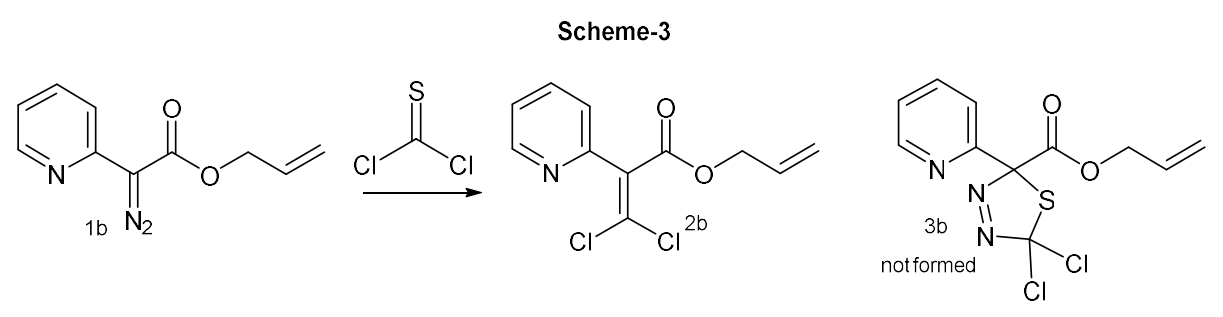

After getting disubstituted diazo results, now treated with mono substituted diazo derivatives, ethyl diazo acetate 1c with thiophosgene. Isolated ethyl 5-chloro-1,3,4thiadiazole-2-carboxylate $\mathbf{2 c}$, as a major one along with minor dichloro $\mathbf{3 c}$, where the dichloro bearing carbon shows at $5.2 \mathrm{ppm}$ in Proton-NMR and 103 ppm in carbon NMR spectra (scheme-4). The characteristic peak of chloro bearing carbon at $157.6 \mathrm{ppm}$ in carbon NMR confirmed the $1,3,4$ thiadiazole ring. It means $\mathbf{3 c}$ is formed first, on 1,4 elimination of $\mathrm{HCl}$ gives $\mathbf{2 c}$. In reported method, 1,3,4-thaizdiazole-2-carboxylate were isolated as single by using thiolate ${ }^{16}$. or by using thiophosgene ${ }^{17}$ gives mixture or products, ethyl 5-chloro-1,3,4-thiadiazole-2-carboxylate 2c along with ethyl 5-chloro1,3,4-thiadiazole-2-carboxylate $\mathbf{3 d}$.

\section{Scheme-4}
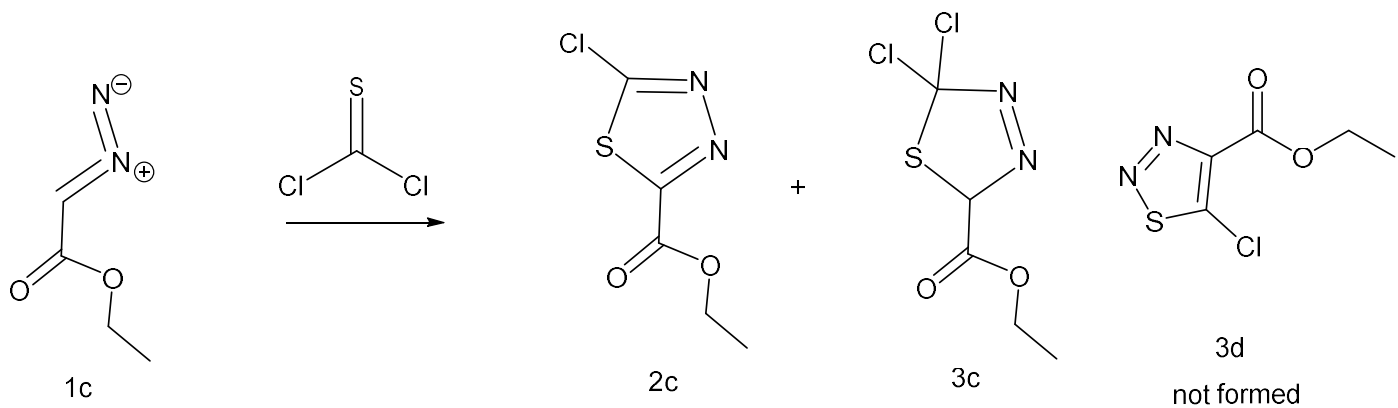

Bromo version, $\mathrm{CSBr}_{2}$ were prepared by treating thiophosgene ${ }^{18}$ with lithium bromide in ethyl acetate at $70^{\circ} \mathrm{C} . \mathrm{FTIR}^{19}$ shows slight change in frequency. This $\mathrm{CSBr}_{2}$ is not much explored in organic synthesis ${ }^{7}$. When $\mathrm{CSBr}_{2}$ was treated with diazo $1 \mathrm{a}$, instead of olefin halo derivatives, it gives dibromo product 4 a exclusively. Mass $M / z$ shows 532 , which is consistent with molecular formula 525 and on combination with Lithium ion. The carbon 
peaks of dibromo bearing carbon in NMR data (58ppm) is matching with predicted value. The Proton NMR data is consistent with reported values ${ }^{20}$ where $4 a$ are prepared by liquid bromine. The sulfone ${ }^{21}$ version of diazo $1 \mathrm{~d}$ gives its dibromo $\mathbf{4 d}$ exclusively. The spectral data is consistent with reported method ${ }^{22}$.

\section{Scheme-5}

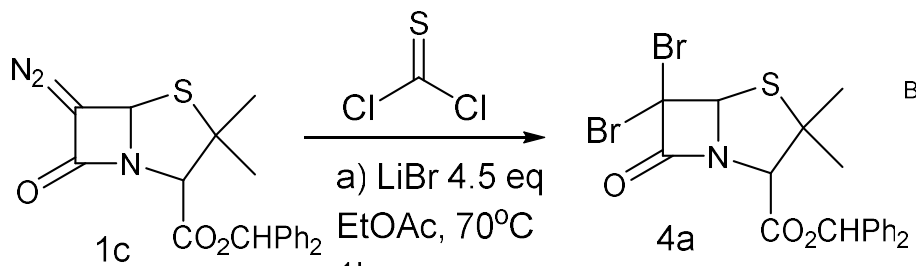
$1 \mathrm{~h}$,

b) $r t, 12 h$<smiles>CC1(C)C(C(=O)O)N2C(=O)C(=N)C2[SH]1(=O)O</smiles>

b) $\mathrm{rt}, 12 \mathrm{~h}$

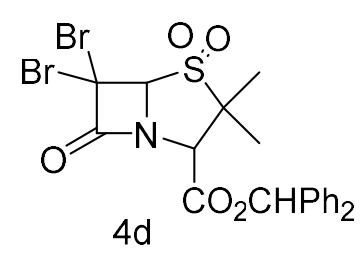

$4 d$<smiles>CC1(C)SC2C(=O)C3(N=NC(Br)(Br)S3)C(=O)N2C1C(=O)O</smiles>
$5 a$ not formed<smiles>CC1(C)C(C(=O)OCc2ccccc2)N2C(=O)C3(N=NC(Br)(Br)S3)C2S1(=O)=O</smiles>

not formed<smiles>CC1(C)SC2C(=C(Br)Br)C(=O)N2C1C(=O)OCc1ccccc1</smiles>

not formed

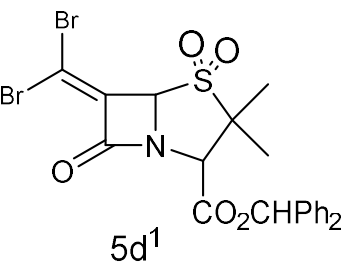

not formed

The pyridine diazo $\mathbf{1 b}$, formed diazine $\mathbf{4 b}$. Traditionally diazirine conversions occurs at room temperature in $\mathrm{THF}^{23}$ and by irradiation ${ }^{24}$. The $\mathbf{4 b}$ shows bromine addition occurs on allyl bond, the mass $\mathrm{m} / \mathrm{z}$ shows $370[\mathrm{M}+(363)+$ lithium ion (7)], and FT-IR further confirmed the $\mathbf{4 b}$ formation. (scheme-6)<smiles>C=CCOC(=O)C(=N)c1ccccn1</smiles>
$1 \mathrm{~b}$ $\underset{\text { a) } \mathrm{LiBr} 4.5 \mathrm{eq}}{\stackrel{\mathrm{Cl}}{\stackrel{\mathrm{ClOAC}}{ } 70^{\circ} \mathrm{C}}}$

$1 \mathrm{~h}$,

b) rt, $12 \mathrm{~h}$
Scheme-6<smiles>O=C(OCC(Br)CBr)C1(c2ccccn2)N=N1</smiles><smiles>C=CCOC(=O)C1(c2ccccn2)N=NC(Br)(Br)S1</smiles><smiles>C=CCOC(=O)C(=C(Br)Br)c1ccccn1</smiles> 
On treatment with mono substituted diazo 1c, we isolated the exclusively ethyl 5-bromo1,3,4-thiadiazole-2-carboxylate $\mathbf{4 c}$. The NMR value are consistent with reported values ${ }^{25-}$ ${ }^{26}$ where it synthesized by using Sandmeyer reaction The structure is further confirmed by single crystal data (hard copy only). The ethyl 5-bromo-1,2,3-thiadiazole-2-carboxylate 5c was not formed under this reaction conditions. (Scheme-7)

Scheme-7
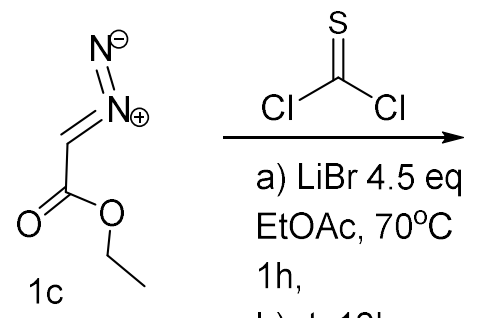

a) $\mathrm{LiBr} 4.5 \mathrm{eq}$ EtOAc, $70^{\circ} \mathrm{C}$ $1 \mathrm{~h}$,

b) $\mathrm{rt}, 12 \mathrm{~h}$<smiles>CCOC(=O)c1nnc(Br)s1</smiles>

$4 \mathrm{c}$<smiles>CCOC(=O)c1nnsc1Br</smiles>

not formed

The plausible explanation is, that we used excess (4.5 equivalent) of lithium bromide for preparation of $\mathrm{CSBr}_{2}$. In case of disubstituted diazo, the bromide ion is more reactive then $\mathrm{CSBr}_{2}$, to give the gem-dibromo product, whereas in mono substituted diazo reacted with $\mathrm{CSBr}_{2}$ and gives the expected 5 membered ring. $\mathrm{CSBr}_{2}$ is indeed formed in the reaction, it incorporated in product $\mathbf{4 c}$.

\begin{tabular}{|c|c|c|}
\hline Substrate. & CSBr 2. & Bromide ion \\
\hline Disubstituted diazo, $1 \mathrm{a}, 1 \mathrm{~b}, 1 \mathrm{~d}$, & No & Yes \\
\hline Mono substituted diazo, $1 \mathrm{c}$ & Yes & No \\
\hline
\end{tabular}

lodo version, $\left(\mathrm{CSI}_{2}\right)$ were prepared by treating thiophosgene ${ }^{27-28}$, FTIR shows slight change in frequency. When iodo treated with diazo $1 \mathrm{a}$, as expected it give diiodo products $6 a$. Mass $M / z$ shows 625 , which is consistent with molecular formula 618 and on combination with Lithium ion. The carbon peaks of diiodo bearing carbon in NMR data (-7 ppm) is matching with predicted value. This method is addition to literature, where in reported method 6 a obtained one out of multiple products ${ }^{15}$. (Scheme-8) 


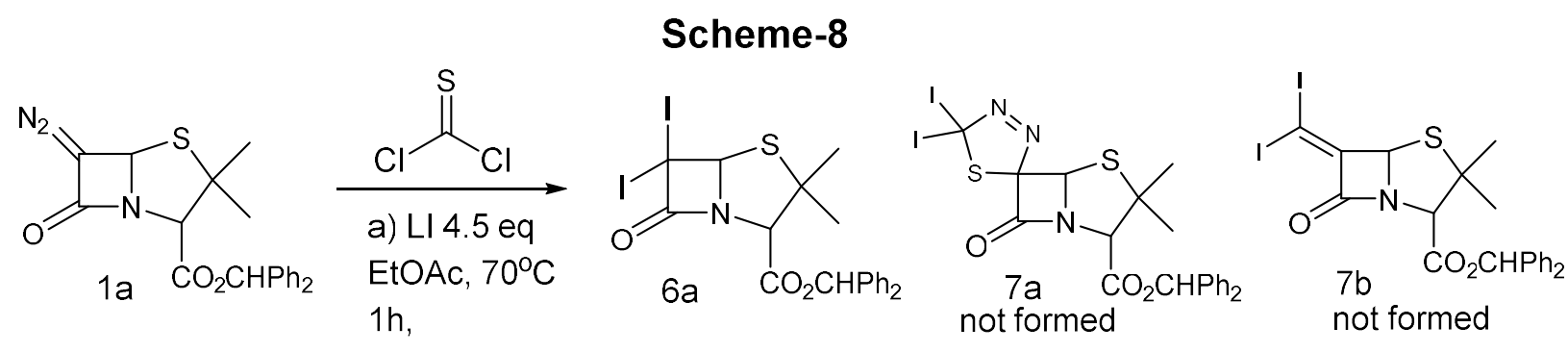

b) rt, $12 h$

On treatment with ethyl diazo acetate $1 \mathrm{c}$, we isolated the diiodo $\mathbf{6 c}$ product, where diazo displaced by diiodo, there is no cyclic product $7 \mathrm{c}$ were isolated. Independently the 7c were prepared by using iodine metal were reported 29,30

\section{Scheme-9}

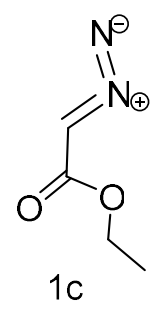

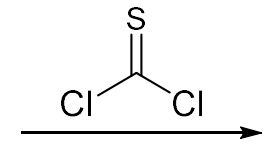

a) LI $4.5 \mathrm{eq}$ EtOAc, $70^{\circ} \mathrm{C}$ $1 \mathrm{~h}$,

b) rt, $12 \mathrm{~h}$<smiles>CCOC(=O)C(I)I</smiles>
$6 c$<smiles>CCOC(=O)c1nnc(I)s1</smiles>

not formed

The plausible explanation is, that we used excess ( 4.5 equivalent) of lithium iodide for preparation of $\mathrm{CSI}_{2}$, irrespective of substitution on diazo substrate, the lodide ion is more reactive then $\mathrm{CSI}_{2}$, to give the gem-diiodo product.

\begin{tabular}{|c|c|c|}
\hline Substrate. & CSI 2. & Iodide ion \\
\hline Disubstituted diazo & No & Yes \\
\hline Mono substituted diazo & No & Yes \\
\hline
\end{tabular}

Typical Procedure: To a solution of thiophosgene $(1.0 \mathrm{eq})$ in ethyl acetate $(25-50 \mathrm{ml})$ in a single neck round bottom flask, added lithium bromide $(4.5 \mathrm{eq})$ of and heated at $70^{\circ} \mathrm{C}$ for $1 \mathrm{~h}$. Cool to room temperature, then added diazo $(0.8 \mathrm{eq})$ and stir at room temperature for overnight. After reaction complete (checked by TLC), extracted with ethyl acetate (50 $\mathrm{mL})$, washed with water $(50 \mathrm{~mL})$, brine solution $(50 \mathrm{~mL})$, dried over anhydrous $\mathrm{MgSO}_{4}$ 
and filter. All volatiles were removed, and the residue was purified using column chromatography (EtOAc:hex) to give products in $55-78 \%$ yield.

\begin{tabular}{|c|c|c|c|c|}
\hline Products & Dihalo-alkene & Gem-Dihalo & 1,3,4-Thiazole & Addition to olefin \\
\hline \multicolumn{5}{|c|}{ Diazo with thiophosgene } \\
\hline $2 \mathrm{a}$ & Major & & Minor & \\
\hline $2 b$ & Product & & & \\
\hline $2 \mathrm{c}$ & & & Product & \\
\hline \multicolumn{5}{|c|}{ Diazo with Bromo-thiophosgene and Bromide ion } \\
\hline $4 \mathrm{a}$ & & Product & & \\
\hline $4 \mathrm{~b}$ & & & & Product \\
\hline $4 \mathrm{c}$ & & & Product & \\
\hline $4 \mathrm{~d}$ & & Product & & \\
\hline \multicolumn{5}{|c|}{ Diazo with iodo-thiophosgene and Iodide ion } \\
\hline $6 \mathrm{a}$ & & Product & & \\
\hline $6 \mathrm{c}$ & & Product & & \\
\hline
\end{tabular}

In conclusion, the product formation is depending on substituted diazo substrate, in case

of substrate $\mathbf{1 a}, \mathbf{1 d}$, the results are consistent with dihalo substitution, whereas as in case pyridine 1c, diazine is formed and bromine addition to allyl bond occurs. In case mono substituted diazo substrate $\mathbf{1 b}$, cyclic 1,2,3-thiadiazoles are formed, except in case iodo version simple substitution occurs.

\section{EXPERIMENTAL PART}

All chemicals and other materials were reagent grade unless otherwise specified. All reactions were carried out in a dry nitrogen atmosphere. ${ }^{1} \mathrm{H}$ and ${ }^{13} \mathrm{C}$ NMR spectra were obtained on a 300-MHz Bruker Avance NMR spectrometer. All chemicals' shifts are reported in parts per millions. Infrared spectra were obtained on a Nicolet Magna-IR 560 spectrometer E.S.P. Melting points were collected on a TA Instruments DSC 2010 
Differential Scanning Calorimeter using a heating rate of $10^{\circ} \mathrm{C} / \mathrm{min}$ and nitrogen as a purge gas.

Benzhydryl

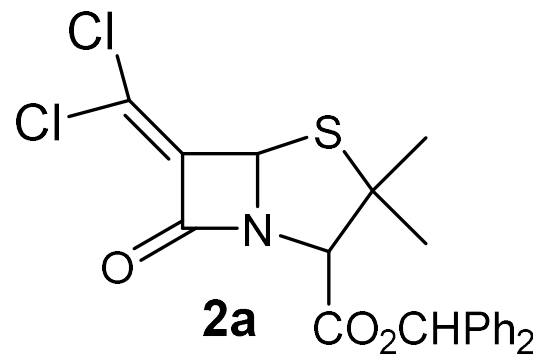

6-(dichloromethylene)-3,3-dimethyl-7-oxo-4-thia-1azabicyclo[3.2.0]heptane-2-carboxylate (2a): ${ }^{1} \mathrm{H}-\mathrm{NMR}$ (300 MHz, CDCl 3$):{ }^{\delta} 7.32(\mathrm{~d}, 10 \mathrm{H}), 6.93(\mathrm{~s}, 1 \mathrm{H}), 5.64(\mathrm{~s}$, $1 \mathrm{H}), 4.50(\mathrm{~s}, 1 \mathrm{H}), 1.49(\mathrm{~s}, 3 \mathrm{H}), 1.25(\mathrm{~s}, 3 \mathrm{H}) .{ }^{13} \mathrm{C}-\mathrm{NMR}(75.0$ $\left.\mathrm{MHz}, \mathrm{CDCl}_{3}\right):{ }^{\circ} 168.1,165.7,1.38 .7,138.6,138.5,128.6$ $128.5,128.4,128.2,127.5,127.2,126.8,78.6,69.9,64.8,33.2,25.7$.

\section{Benzhydryl 2-(1,1-dichloro-3-oxoprop-1-en-2-yl)-5,5-dimethyl-4,5-dihydrothiazole- Cl

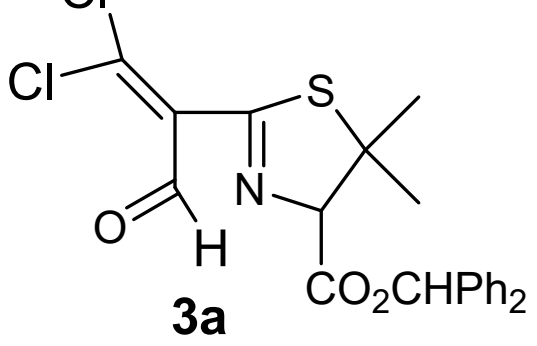 \\ 4-carboxylate (3a): ${ }^{1} \mathrm{H}-\mathrm{NMR}\left(300 \mathrm{MHz}^{\mathrm{C}} \mathrm{CDCl}_{3}\right)$ : ${ }^{\delta} 9.80$ (s, 1H), $7.16(\mathrm{~s}, 10 \mathrm{H}), 6.80(\mathrm{~s}, 1 \mathrm{H}), 5.05(\mathrm{~s}, 1 \mathrm{H}), 144-1.35$ (d, 6H). ${ }^{13} \mathrm{C}-\mathrm{NMR}\left(75.0 \mathrm{MHz}, \mathrm{CDCl}_{3}\right): \delta 186.8,165.0,138.7$, $128.6,128.5,128.4,128.2,127.5,127.0,79.5,65.6,53.6$}

24.6. Mass m/z: 000.00. IR (KBr) (wavenumber, $\mathrm{cm}^{-1}$ ): 3062 (C-Cs), 2061 (S-C=N), 1744 $(\mathrm{H}-\mathrm{C}=\mathrm{O}), 1670(\mathrm{O}-\mathrm{C}=\mathrm{O}), 1495,1453$ (C=Cs), 1193 (C-Cl), 952 (C-Cb). Mass m/z: (M+), 447.2 observed.

allyl 3,3-dichloro-2-(pyridin-2-yl) acrylate (2b): ${ }^{1} \mathrm{H}-\mathrm{NMR}\left(300 \mathrm{MHz}^{\mathrm{C}} \mathrm{CDCl}_{3}\right):{ }^{\delta} 8.92$ (s,<smiles>C=CCOC(=O)C(=C(Cl)Cl)c1ccccn1</smiles>
$1 \mathrm{H}), 8.25-8.23(\mathrm{~d}, 1 \mathrm{H}, J=5.6 \mathrm{~Hz}), 7.62(\mathrm{~s}, 1 \mathrm{H}), 7.30$ (s, $1 \mathrm{H}), 7.13-6.11(\mathrm{t}, 1 \mathrm{H}, J=2.45 \mathrm{~Hz}), 5.51-5.47(\mathrm{~d}, 1 \mathrm{H}, \mathrm{J}=$ 12.86 Hz), 5.35-5.33 (s, 1H, J = 7.41 Hz), 4.98 (s, 2H). ${ }^{13} \mathrm{C}-\mathrm{NMR}\left(75.0 \mathrm{MHz}, \mathrm{CDCl}_{3}\right): \delta 160.3,134.4,131.4$ $129.1,128.4,125.5,118.4,118.3,116.2,64.9 . \mathrm{IR}(\mathrm{KBr})\left(\right.$ wavenumber, $\left.\mathrm{cm}^{-1}\right): 3100(\mathrm{C}-$ 
Cs), 1719 (O-C=O), 1515, 1437 (C=Cs), 1193 (C-Cl), 934 (C-Cb). Mass m/z (M-57+Li) 210 observed.

Ethyl 5-chloro-1,3,4-thiadiazole-2-carboxylate ${ }^{16}$ (2c): ${ }^{1} \mathrm{H}-\mathrm{NMR}\left(400 \mathrm{MHz}, \mathrm{CDCl}_{3}\right):{ }^{\delta}$ $\mathrm{Cl}$<smiles>CCOC(=O)c1nnc(C)s1</smiles>
4.55-4.50 (q, 2H, J=5.35 Hz), 1.49-1.25 (t, 3H, J = 5.37 Hz). ${ }^{13} \mathrm{C}-\mathrm{NMR}$ (75.0 MHz, $\left.\mathrm{CDCl}_{3}\right):{ }^{\circ}$ 162.1, 158.6, 157.6, 63.7, 14.0.

Ethyl 5,5-dichloro-2,5-dihydro-1,3,4-thiadiazole-2-carboxylate (3c): ${ }^{1} \mathrm{H}-\mathrm{NMR}$ (400<smiles>CCOC(=O)C1N=NC(Cl)(Cl)S1</smiles>
$\left.\mathrm{MHz}, \mathrm{CDCl}_{3}\right):{ }^{\delta} 5.29(\mathrm{~s}, 1 \mathrm{H}), 4.28-4.17(\mathrm{~m}, 2 \mathrm{H}), 1.31-1.23(\mathrm{~m}, 3 \mathrm{H}) .{ }^{13} \mathrm{C}-$ $\operatorname{NMR}\left(75.0 \mathrm{MHz}, \mathrm{CDCl}_{3}\right):{ }^{\overline{0}} 165.8,103.4,63.6,48.9,13.9$.

Carbonothioic dibromide (): To a solution of $2.0 \mathrm{~g}(17.3 \mathrm{mmol})$ of thiophosgene in ethyl $\mathrm{S}$ acetate $(50 \mathrm{ml})$ in a single neck round bottom flask, added $6.8 \mathrm{~g}$ of $(4.4 \mathrm{eq})$
$\mathrm{Br}$ of lithium bromide (Mol.Wt $87.112 \mathrm{~g} / \mathrm{mol})$ and heated at $70^{\circ} \mathrm{C}$ for $1 \mathrm{~h}$. Cool to room temperature and used in next reactions. IR $(\mathrm{KBr})$ (wavenumber, $\left.\mathrm{cm}^{-1}\right): 1635$ $(\mathrm{C}=\mathrm{S}), 1262(\mathrm{Br}-\mathrm{C})$, 1503, $1098(\mathrm{C}-\mathrm{Cb})$. 
benzhydryl 6,6-dibromo-3,3-dimethyl-7-oxo-4-thia-1-azabicyclo[3.2.0]heptane-2-<smiles>CC1(C)SC2N(C(=O)C2(Br)Br)C1C(=O)O</smiles>

carboxylate ${ }^{22}$ (4a): ${ }^{1} \mathrm{H}-\mathrm{NMR}\left(400 \mathrm{MHz}, \mathrm{CDCl}_{3}\right):{ }^{\delta} 7.38-7.28$ (m, 10H), 6.93 (s. 1H, benzylic), $5.82(\mathrm{~s}, 1 \mathrm{H}, \mathrm{C} 5 \mathrm{CH}), 4.62$ (s, 1H, C3 CH), 1.59 (s, 3H, Me), $1.25(\mathrm{~s}, 3 \mathrm{H}, \mathrm{Me}) \cdot{ }^{13} \mathrm{C}-\mathrm{NMR}$ $\left(100.6 \mathrm{MHz}, \mathrm{CDCl}_{3}\right):{ }^{\delta} \quad 165.5,164.3,138.8,138.7 .128 .54$, 128.52, 128.4, 128.2, 127.3, 126.9, 80.8, 78.6, 69.6, 64.8, 58.6, 33.6, 25.5. IR (KBr) (wavenumber, $\left.\mathrm{cm}^{-1}\right)$ : 3062 (C-Cs), 1783 (O-C=O), 1745 (HN-C=O), 1494, 1452 (C=Cs), 1255 (C-Br), 992 (C-Cb). Mass m/z (M+Li), 532.1 observed.

2,3-dibromopropyl 3-(pyridin-2-yl)-3H-diazirine-3-carboxylate (4b): ${ }^{1} \mathrm{H}-\mathrm{NMR}$ (400<smiles>O=C(OCC(Br)CBr)C1(c2ccccn2)N=N1</smiles>
$\left.\mathrm{MHz}, \mathrm{CDCl}_{3}\right){ }^{\delta} 8.81-8.79(\mathrm{~d}, 1 \mathrm{H}, J=5.2 \mathrm{~Hz}), 8.20-8.18$ $(\mathrm{d}, 1 \mathrm{H}, J=3.68 \mathrm{~Hz}), 7.56-7.52(\mathrm{dd}, 1 \mathrm{H}, J=5.2 \& 0.5 \mathrm{~Hz})$, 7.18-7.16 (t, $1 \mathrm{H}, J=6.0 \mathrm{~Hz}), 4.82(\mathrm{~m}, 2 \mathrm{H}), 4.50-4.47(\mathrm{~m}$, 1H), 3.85-3.80 (m, 2H). ${ }^{13} \mathrm{C}-\mathrm{NMR}\left(75.0 \mathrm{MHz}, \mathrm{CDCl}_{3}\right):{ }^{\delta}$ 160.0, 134.9, 129.7, 128.2, 125.9, 118.9, 116.5, 65.9, 45.9, 32.3. IR ( $\mathrm{KBr})$ (wavenumber, $\left.\mathrm{cm}^{-1}\right): 3103$ (C-Cs), $1722(\mathrm{O}-\mathrm{C}=\mathrm{O})$, 1514, 1434 (C=Cs), 1198 (C-Br), 981 (C-Cb). Mass m/z: (M+Li) 370.1 observed.

Ethyl 5-bromo-1,3,4-thiadiazole-2-carboxylate ${ }^{26}$ (4c): ${ }^{1} \mathrm{H}$ NMR $\left(300 \mathrm{MHz}, \mathrm{CDCl}_{3}\right) \delta$ (N) 4.56-4.51 (q, $2 \mathrm{H}, J=5.32 \mathrm{~Hz}), 1.48-1.45(\mathrm{~d}, J=5.33 \mathrm{~Hz}, 3 \mathrm{H}) .{ }^{13} \mathrm{C} \mathrm{NMR}$ $\left(75.0 \mathrm{MHz}, \mathrm{CDCl}_{3}\right) \delta 163.5,157.3,144.6,63.6,13.9$. IR $(\mathrm{KBr})$ 4c (wavenumber, $\left.\mathrm{cm}^{-1}\right): 2986$ (C-Cs), $1749(\mathrm{O}-\mathrm{C}=\mathrm{O}), 1473,1452$ (C=Cs), 1266 (C-Br), 1030 (C-Cb). Mass m/z: (M+Li) 242.9 observed 
benzhydryl 6,6-dibromo-3,3-dimethyl-7-oxo-4-thia-1-azabicyclo[3.2.0]heptane-2-<smiles>CC1(C)C(C(=O)Oc2ccccc2)N2C(=O)C(Br)(Br)C2S1(=O)=O</smilescarboxylate 4,4-dioxide ${ }^{22}$ (4d): ${ }^{1} \mathrm{H}-\mathrm{NMR}\left(300 \mathrm{MHz}, \mathrm{CDCl}_{3}\right)$ : б 7.35-7.34 (m, 10H), 6.96 (s. 1H), $4.99(\mathrm{~s}, 1 \mathrm{H}), 4.60$ (s, 1H), $1.58(\mathrm{~s}, 3 \mathrm{H}), 1.12(\mathrm{~s}, 3 \mathrm{H})$.

Carbonothioic diiodide (): To a solution of $1.0 \mathrm{~g}(8.65 \mathrm{mmol})$ of thiophosgene in ethyl acetate $(25 \mathrm{ml})$ in a single neck round bottom flask, added $5.2 \mathrm{~g}$ of $(4.4 \mathrm{eq})$ of
lithium lodide (Mol.Wt $133.84 \mathrm{~g} / \mathrm{mol}$ ) and heated at $70^{\circ} \mathrm{C}$ for $1 \mathrm{~h}$. Cool to room temperature and used in next reactions. IR $(\mathrm{KBr})$ (wavenumber, $\left.\mathrm{cm}^{-1}\right)$ : $1594(\mathrm{C}=\mathrm{S}), 1270$ (I-C), 1503, 1044 (C-Cb).

\section{benzhydryl 6,6-diiodo-3,3-dimethyl-7-oxo-4-thia-1-azabicyclo[3.2.0]heptane-2-}

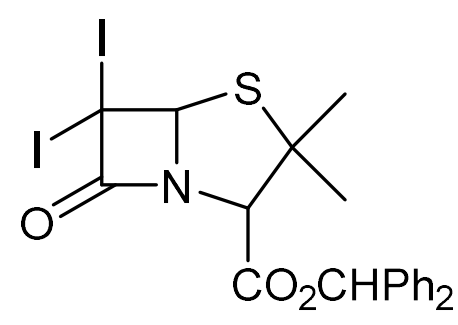
carboxylate (6a): ${ }^{1} \mathrm{H}-\mathrm{NMR}\left(400 \mathrm{MHz}, \mathrm{CDCl}_{3}\right):{ }^{\delta} 7.36-7.28(\mathrm{~m}$, 10H), 6.92, (s, 1H), $5.80(\mathrm{~s}, 1 \mathrm{H}), 4.62(\mathrm{~s}, 1 \mathrm{H}), 1.60(\mathrm{~s}, 3 \mathrm{H}), 1.25$ (s, 3H). ${ }^{13} \mathrm{C}-\mathrm{NMR}\left(100.6 \mathrm{MHz}, \mathrm{CDCl}_{3}\right):$ б 167.3, 165.7, 138.9, 138.8, 81.7, 78.6, 70.2, 65.8, 34.0, 26.3, -7.46 (C-I). IR (KBr) (wavenumber, $\left.\mathrm{cm}^{-1}\right)$ : 3055 (C-Cs), $1787(\mathrm{O}-\mathrm{C}=\mathrm{O}), 1745(\mathrm{HN}-\mathrm{C}=\mathrm{O}), 1495,1453(\mathrm{C}=\mathrm{Cs})$, 1265 (C-I), 738 (C-Cb). Mass m/z: (M+Li) 625.9 observed.

Ethyl 2,2-diiodoacetate ${ }^{30}$ (6c): ${ }^{1} \mathrm{H}$ NMR $\left(300 \mathrm{MHz}, \mathrm{CDCl}_{3}\right)^{\delta} 5.34$ (s, 1H), 4.31-4.24 (q,

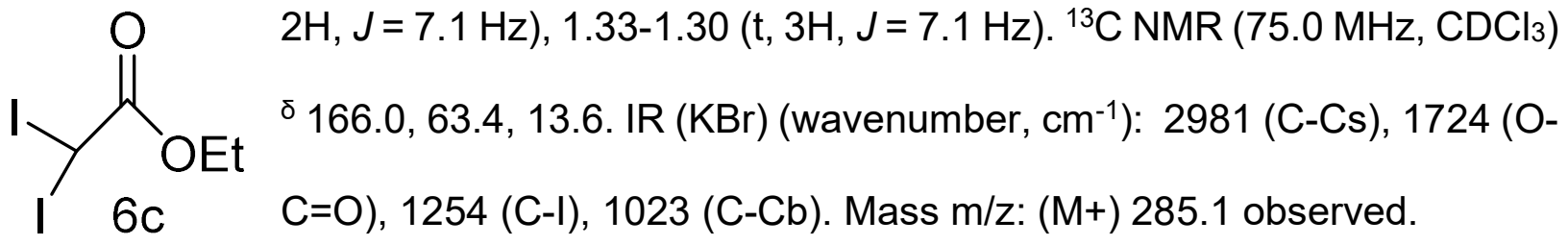


Page 12 of 13

\section{ACKNOWLEDGMENTS}

S.R.G is thankful to SMU, Chemistry Department for supporting from September-2005 to

December-2005.

\section{Reference:}

1. Tilles, H., Thiophosgene. Org. Sulfur Compounds. 1966, 2, 311-36.

2. McMurry, J. E.; Haley, G. J.; Matz, J. R.; Clardy, J. C.; Van Duyne, G.; Gleiter, R.; Schaefer, W.; White, D. H., Tetracyclo[8.2.2.22,5.26,9]octadeca-1,5,9-triene. Journal of the American Chemical Society 1984, 106 (17), 5018-5019.

3. Rall, K.; Sundermeyer, W., Synthesis of perhalogenated 1,3-dithietane S-oxides and thiiranes. J. Fluorine Chem. 1990, 47 (1), 121-30.

4. Thijs, L.; Strating, J.; Zwanenburg, B., Chemistry of sulfines. XIV. Benzo[b]thiophene derivatives from dichlorosulfine and diaryldiazomethanes. Recl. Trav. Chim. Pays-Bas 1972, 91 (11), 1345-51.

5. Demaree, P.; Doria, M.-C.; Muchowski, J. M., The reaction of certain $\alpha$-diazocarbonyl compounds with thiophosgene and ethyl chlorodithioformate. Canadian Journal of Chemistry 1977, 55 (2), 243-250.

6. Bushby, R. J.; Pollard, M. D., The introduction of alkylidene substituents into the 4-position of the $3,3,5,5$-tetramethyl- $\Delta 1$-pyrazoline nucleus by the thioketone plus diazo alkane reaction. Synthesis of tetrasubstituted episulfides and alkenes. J. Chem. Soc., Perkin Trans. 1 1979, (10), 2401-8.

7. Sato, S.; Furukawa, N., Thiocarbonic acids and derivatives. Sci. Synth. 2005, 18, 821-968.

8. Luo, H.; Zhou, H. Dihydropyridophthalazinone derivative, and preparation method therefor and application thereof. CN110272419A, 2019.

9. Balasubramanian, G.; Paul-Satyaseela, M.; Srinivasan, C. V.; Lyer, S. R.; Periasamy, H.; Parameswaran, V.; Thirunavukkarasu, B.; Gunturu, P. R.; Deshkumar, M. D.; Jakkala, V.; Nargund, R. P.; Miller, M.; Singh, S.; Dong, S.; Wang, H.; Young, K. Preparation of carbapenem compounds for the treatment of bacterial infections. WO2017158616A1, 2017.

10. Buynak, J. D.; Rao, A. S.; Adam, G. C. Preparation of 7-alkylidene cephalosporins to inhibit elastase activity. US5760027A, 1998.

11. Hu, Y.-h.; Hu, Y.-m., New process for the synthesis of tazobactam. a $\beta$-lactamase inhibitor. Hecheng Huaxue 2003, 11 (3), 243-245.

12. Lee, J. M.; Yim, C. B.; Im, C., Synthesis of 7-arylidene cephalosporanates for $\beta$-lactamase inhibitor. Yakhak Hoechi 2008, 52 (4), 311-315.

13. Buynak, J. D.; Vogeti, L. Preparation of 7-alkylidene-3-substituted-3-cephem-4-carboxylates as $\beta$ lactamase inhibitors. WO2003020732A2, 2003.

14. Buynak, J. D.; Chen, H.; Vogeti, L.; Gadhachanda, V. R.; Buchanan, C. A.; Palzkill, T.; Shaw, R. W.; Spencer, J.; Walsh, T. R., Penicillin-derived inhibitors that simultaneously target both metallo- and serine$\beta$-lactamases. Bioorg. Med. Chem. Lett. 2004, 14 (5), 1299-1304.

15. Micetich, R. G.; Maiti, S. N.; Tanaka, M.; Yamazaki, T.; Ogawa, K., Studies on 6-halo- and 6,6dihalopenicillins: the rearrangement of methyl 6,6-dibromopenicillanate to 1,4-thiazepine. J. Org. Chem. 1986, 51 (6), 853-8.

16. Du, W.; Wen, K.; Fu, Y.; Lv, H.; He, J.; Qin, D.; Li, Y.; Duan, J.; Li, Y.; Ai, C.; Tu, Z.; Chen, Y.; Li, X. A class of bifunctional chimeric heterocyclic compounds for targeted degradation of androgen receptors and application. WO2020211822A1, 2020.

17. Gray, N. S.; Zhou, W. Preparation of EGFR modulators for treating or preventing kinase-mediated disorders. WO2011079231A1, 2011. 
18. Keinan, E.; Sahai, M., Diiodosilane. 3. Direct synthesis of acyl iodides from carboxylic acids, esters, lactones, acyl chlorides and anhydrides. The Journal of Organic Chemistry 1990, 55 (12), 3922-3926.

19. Tobón, Y. A.; Romano, R. M.; Della Védova, C. O.; Downs, A. J., Formation of New Halogenothiocarbonylsulfenyl Halides, $X C(S) S Y$, through Photochemical Matrix Reactions Starting from $\mathrm{CS} 2$ and a Dihalogen Molecule XY (XY = Cl2, Br2, or BrCl). Inorganic Chemistry 2007, 46 (11), 4692-4703.

20. Buynak, J. D.; Chen, H. Preparation of penicillin derivatives as inhibitors of serine and metallo- $\beta$ lactamases. WO2003087105A1, 2003.

21. Sandanayaka, V. P.; Prashad, A. S.; Yang, Y.; Williamson, R. T.; Lin, Y. I.; Mansour, T. S., Spirocyclopropyl $\beta$-Lactams as Mechanism-Based Inhibitors of Serine $\beta$-Lactamases. Synthesis by Rhodium-Catalyzed Cyclopropanation of 6-Diazopenicillanate Sulfone. J. Med. Chem. 2003, 46 (13), 25692571.

22. Totir, M. A.; Helfand, M. S.; Carey, M. P.; Sheri, A.; Buynak, J. D.; Bonomo, R. A.; Carey, P. R., Sulbactam Forms Only Minimal Amounts of Irreversible Acrylate-Enzyme with SHV-1 $\beta$-Lactamase. Biochemistry 2007, 46 (31), 8980-8987.

23. Heydt, H., Product class 21: diazo compounds. Sci. Synth. 2004, 27, 843-935.

24. Bogdanova, A.; Popik, V. V., Experimental and Theoretical Investigation of Reversible Interconversion, Thermal Reactions, and Wavelength-Dependent Photochemistry of Diazo Meldrum's Acid and its Diazirine Isomer, 6,6-Dimethyl-5,7-dioxa-1,2-diaza-spiro[2,5]oct-1-ene-4,8-dione. J. Am. Chem. Soc. 2003, 125 (46), 14153-14162.

25. Gurusamy, R.; Murugan, S.; Sarangthem, R.; Madhavrao, K. M.; Jhala, V. S.; Ashamoni, S.; Khan, U.; Ebhad, D. L.; Verma, A. K.; Garg, R.; Venkatesha, H. M.; Klausener, A. G. M. Preparation of nitrone compounds for protecting crops. WO2020095161A1, 2020.

26. Schafer, G.; Fleischer, T.; Ahmetovic, M.; Abele, S., Development of a Scalable Route for a Key Thiadiazole Building Block via Sequential Sandmeyer Bromination and Room-Temperature Suzuki-Miyaura Coupling. Org. Process Res. Dev. 2020, 24 (2), 228-234.

27. Stuedel, R., Reaction of carbon monosulfide and monoselenide with chalcogens and halogens. Angew. Chem., Int. Ed. Engl. 1967, 6 (7), 635.

28. Lyashchuk, S. N.; Vasil'eva, N. V.; Skrypnik, Y. G., Theoretical study of the structure, electronic state and reactivity of halo-substituted sulfines and sulfenes. Zh. Org. Khim. 1996, 32 (4), 509-515.

29. Tang, S.; Gao, X.; Lei, A., Decarboxylative (4+1) Oxidative Annulation of Malonate Monoesters with 2-Vinylpyridine Derivatives. Adv. Synth. Catal. 2016, 358 (18), 2878-2882.

30. Li, P.; Zhao, J.; Shi, L.; Wang, J.; Shi, X.; Li, F., lodine-catalyzed diazo activation to access radical reactivity. Nat. Commun. 2018, 9 (1), 1-9. 